\title{
Diagnostic values of serum tumor markers Cyfra2 I-I, SCCAg, ferritin, CEA, CAI9-9, and AFP in oral/oropharyngeal squamous cell carcinoma
}

This article was published in the following Dove Press journal:

OncoTargets and Therapy

3 June 2016

Number of times this article has been viewed

Chuanshu Yuan

Kai Yang

Hong Tang

Dan Chen

Department of Oral and Maxillofacial Surgery, the First Affiliated Hospital of Chongqing Medical University, Chongqing, People's Republic of China
Background: At present, the research on serum tumor markers in the early diagnosis of malignant tumors has aroused widespread concern. The aim of this study was to investigate the diagnostic values of serum tumor markers cytokeratin 19 fragment (Cyfra21-1), squamous cell carcinoma antigen (SCCAg), ferritin, carcinoembryonic antigen (CEA), carbohydrate antigen 19-9 (CA19-9), and $\alpha$-fetoprotein (AFP) for patients with oral/oropharyngeal squamous carcinoma (OSCC/OPSCC).

Methods: One hundred and sixty-nine cases of patients with OSCC/OPSCC as the experimental group, 86 cases of oral benign tumor patients as the control group, and 30 cases of healthy people as the normal control group were studied. The levels of serum Cyfra21-1, SCCAg, ferritin, CEA, CA19-9, and AFP were measured using electrochemiluminescence immunoassay.

Results: The levels of serum Cyfra21-1, SCCAg, ferritin, and CEA in patients with OSCC/ OPSCC were significantly higher than those of benign tumor and healthy control group $(P<0.05)$. The levels of CA19-9 and AFP showed no significant difference between patients with OSCC/ OPSCC, benign tumor, and healthy group $(P>0.05)$. The level of serum Cyfra21-1 in patients with early OSCC/OPSCC (stage I + II) was significantly higher than that of benign tumor and healthy control group $(P<0.05)$. However, the levels of serum SCCAg, ferritin, CEA, CA19-9, and AFP showed no significant difference between patients with early OSCC/OPSCC, benign tumor, and healthy control group $(P>0.05)$. The levels of serum Cyfra21-1, SCCAg, ferritin, and CEA in the middle-late stage of patients with OSCC/OPSCC (stage III + IV) were significantly higher than those of patients with the early OSCC/OPSCC, benign tumor, and healthy control group $(P<0.05)$. The diagnostic cutoff levels of Cyfra21-1, SCCAg, ferritin, and CEA were 2.17, 0.72, 109.95, and $1.99 \mathrm{ng} / \mathrm{mL}$, respectively. The sensitivities were $60.36 \%, 73.37 \%, 81.66 \%$, and $66.27 \%$, respectively. The specificities were $81.03 \%, 68.10 \%, 40.52 \%$, and $61.21 \%$, respectively.

Conclusion: Cyfra21-1, SCCAg, ferritin, and CEA had diagnostic values for patients with OSCC/OPSCC. Meanwhile, Cyfra21-1 had better early diagnostic value for patients with OSCC/OPSCC.

Keywords: oral, oropharynx, cancer, tumor marker, diagnosis

\section{Introduction}

Oral/oropharyngeal carcinoma accounts for $\sim 2 \%-3 \%$ of the systemic malignant tumor. ${ }^{1}$ Among them, squamous cell carcinoma makes up for $>80 \%$ and in poor prognosis. ${ }^{2-4}$ The overall 5-year survival rate is only $50 \%-60 \%, 5,6$ of which the middle-late 5 -year survival rate is $\sim 20 \%-40 \%,{ }^{1}$ but the early 5 -year survival rate is as high as $80 \%$. ${ }^{1,7}$ Therefore, early diagnosis and treatment are the keys to improve the survival rate and quality of patients with oral/oropharyngeal squamous carcinoma (OSCC/OPSCC). ${ }^{1,2}$ 
In recent years, the research on serum tumor markers (TMs) in the early diagnosis of malignant tumors has aroused widespread concern. ${ }^{7,8}$ The TM is a kind of substance, which may be abnormal because of tumor cells-related gene expression or body response to tumor in the process of the occurrence and proliferation of malignant tumor. ${ }^{9}$ It was reported that the levels of serum TMs showed better diagnostic values in partial malignant tumors, for example, $\alpha$-fetoprotein (AFP) and prostate-specific antigen became the specific TMs of liver cancer and prostate cancer, respectively. ${ }^{10,11}$ It has been demonstrated that squamous cell carcinoma antigen (SCCAg), cytokeratin 19 fragment (Cyfra21-1), and carcinoembryonic antigen (CEA) were significantly higher in cervical squamous cell carcinoma, small cell lung cancer, and colorectal carcinoma respectively; ${ }^{12-14}$ the levels of serum Cyfra21-1 and SCCAg were significantly higher in partial patients with head and neck squamous cell carcinoma, ${ }^{15,16}$ suggesting that these serum TMs might have better clinical value in the screening and diagnosis of the tumor. So far, there is still a lack of any simple or effective method to screen and diagnose patients with OSCC/OPSCC. Since the levels of the serum TMs are different among different malignant tumors, six kinds of serum TMs which were commonly used in clinics at present, including Cyfra21-1, SCCAg, ferritin, CEA, carbohydrate antigen 19-9 (CA19-9), and AFP were selected for this study. ${ }^{10,17-20}$ The aim of this study was to measure the levels of serum Cyfra21-1, SCCAg, ferritin, CEA, CA19-9, and AFP in newly diagnosed patients with OSCC/OPSCC, to compare them with those of oral benign tumor and healthy persons, to analyze the sensitivities, specificities, and accuracies of various TMs in the diagnosis of patients with OSCC/OPSCC, and to investigate their diagnostic values in patients with OSCC/OPSCC, so as to guide in clinical diagnosis and treatment.

\section{Materials and methods}

\section{Research objects}

Source of patients: One hundred and sixty-nine cases of newly diagnosed patients with OSCC/OPSCC in the Department of Oral and Maxillofacial Surgery in the First Affiliated Hospital of Chongqing Medical University were chosen from January 2013 to January 2015, including 116 males and 53 females. The average age was 53.95 years. The TNM classification and clinical stage were determined based on the 7 th edition of the Union for International Cancer Control standards. ${ }^{15}$ The 169 OSCC/OPSCC cases included 17 cases of stage I, 40 cases of stage II, 61 cases of stage III, and 51 cases of stage IV.
All patients were confirmed by pathological examination, did not receive radiotherapy, chemotherapy, or other treatments. The patients had no malignant tumor in other positions. The data are shown in Table 1. Benign tumor control group: 86 cases of oral benign tumor patients admitted in the hospital were chosen at the same time, including 56 males and 30 females, the average age was 51.35 years. Among them, 25 cases suffered from gingival tumor, 21 cases suffered from papilloma, 20 cases suffered from vascular tumor, 14 cases suffered from benign small salivary gland tumor, and six cases suffered from adamantoblastoma. Healthy control group: 30 cases received the routine physical examination, including 19 males and eleven females; the average age was 51.83 years. They had no systemic disease.

This study was approved by the Biomedicine Ethics Committee of the First Affiliated Hospital of Chongqing Medical University. All patients signed the informed consents.

\section{Methods}

Venous blood samples $(2 \mathrm{~mL})$ were collected from all fasting patients between 7.00 and $8.00 \mathrm{am}$, immediately placed in $5 \mathrm{~mL}$ anticoagulated antisolvent sterile tube (CDRICH Science and Technology, Chengdu, Sichuan, People's Republic of China), and centrifuged (3,500 rpm) for $10 \mathrm{~min}$ utes at room temperature. The serum was separated and measured automatically using electrochemiluminescence immunoassay by Roche Elecsys 2010 analyzer (Roche, Basel, Switzerland).

\section{Statistical analyses}

The data were processed using SPSS 21.0 (IBM Corporation, Armonk, NY, USA) statistical software and accorded with abnormal distribution by Shapiro-Wilk method. The

Table I Clinical conditions of patients with oral/oropharyngeal squamous cell carcinoma

\begin{tabular}{|c|c|c|c|c|c|c|}
\hline \multirow[t]{2}{*}{ Position } & \multicolumn{4}{|c|}{$\begin{array}{l}\text { Clinical } \\
\text { stages }\end{array}$} & \multirow[t]{2}{*}{$\begin{array}{l}\text { Case number } \\
(\%)\end{array}$} & \multirow[t]{2}{*}{$\begin{array}{l}\text { Average age } \\
\text { (years) }\end{array}$} \\
\hline & $\mathbf{I}$ & II & III & IV & & \\
\hline Tongue carcinoma & 10 & 18 & 16 & II & $55(32.54)$ & 54.67 \\
\hline Cheek carcinoma & 3 & 5 & 20 & II & $39(23.08)$ & 52.13 \\
\hline $\begin{array}{l}\text { Oropharyngeal } \\
\text { carcinoma }\end{array}$ & 1 & 6 & 11 & 13 & 31 (I8.34) & 53.61 \\
\hline Mouth floor carcinoma & 2 & 2 & 7 & 6 & $17(10.06)$ & 57.82 \\
\hline Gingival carcinoma & 0 & 5 & 2 & 7 & $14(8.28)$ & 54.29 \\
\hline Palate carcinoma & 0 & 2 & 2 & 3 & $7(4.14)$ & 53.29 \\
\hline Lip carcinoma & 1 & 2 & 3 & 0 & $6(3.55)$ & 49.83 \\
\hline Total & 17 & 40 & 61 & 51 & $169(100)$ & 53.95 \\
\hline
\end{tabular}


measurement data were shown with median and analyzed using Kruskal-Wallis nonparametric rank sum test. The comparison among groups was shown with Mann-Whitney $U$ test. The enumeration data were shown with percentage and tested with $\chi^{2}$ test. The receiver operating characteristic curve analysis was performed. The diagnostic cutoff levels, sensitivities, specificities, and accuracies of various TMs were calculated. Sensitivity $=($ true positive $) /($ true positive + false negative $) \times 100 \%$; specificity $=($ true negative)/(true negative + false positive) $\times 100 \%$; accuracy $=$ (true positive + true negative $) /($ true positive + false negative + true negative + false positive $) \times 100 \% \cdot{ }^{13,21} \mathrm{~A}$ $P$-value of $<0.05$ was considered significant.

\section{Results}

\section{Basic information for patients}

There was no significant difference in the mean age or sex among the three groups $(P>0.05)$. Patients among the three groups were comparable.

\section{Serum levels of Cyfra2I-I, SCCAg, ferritin, CEA, CA 19-9, and AFP in each group}

The levels of serum Cyfra21-1, SCCAg, ferritin, CEA, CA19-9, and AFP in all patients are shown in Table 2. The levels of serum Cyfra21-1, SCCAg, ferritin, and CEA in patients with OSCC/OPSCC were significantly higher than those of the benign tumor group and healthy control group $(P<0.05)$. The benign tumor group and healthy control group showed no significant difference $(P>0.05)$. No significant difference in the levels of serum CA19-9 and AFP was observed among patients with OSCC/OPSCC, benign tumor group, and healthy group $(P>0.05)$.

The patients with OSCC/OPSCC in the early stage (stage I + II) and middle-late stage (stage III + IV) were further compared and analyzed. The result demonstrated that the levels of serum Cyfra21-1, SCCAg, ferritin, and CEA in patients with middle-late OSCC/OPSCC were significantly higher than those of patients with early OSCC/OPSCC, benign tumor group, and healthy control group $(P<0.05)$. In the patients with early OSCC/OPSCC, only the level of serum Cyfra21-1 was significantly higher than that of benign tumor group and healthy control group $(P<0.05)$; the levels of serum SCCAg, ferritin, and CEA showed no significant difference among the three groups $(P>0.05)$. No significant difference in the levels of serum CA19-9 and AFP was observed among patients with early, middle-late OSCC/OPSCC, benign tumor group, and healthy control group ( $P>0.05)$, suggesting that CA19-9 and AFP had no diagnostic value for patients with OSCC/OPSCC.

Table 2 Levels of tumor markers in patients with oral/oropharyngeal squamous cell carcinoma, benign tumor, and healthy control group (M)

\begin{tabular}{|c|c|c|c|c|c|c|}
\hline Groups & $\begin{array}{l}\text { Cyfra2 I-I } \\
\text { (ng/mL) }\end{array}$ & $\begin{array}{l}\text { SCCAg } \\
(n g / m L)\end{array}$ & $\begin{array}{l}\text { Ferritin } \\
(\mathrm{ng} / \mathrm{mL})\end{array}$ & $\begin{array}{l}\text { CEA } \\
(\mathrm{ng} / \mathrm{mL})\end{array}$ & $\begin{array}{l}\text { CAI9-9 } \\
(\mu / m L)\end{array}$ & $\begin{array}{l}\text { AFP } \\
(\mathrm{ng} / \mathrm{mL})\end{array}$ \\
\hline $\begin{array}{l}\text { Oral/oropharyngeal } \\
\text { squamous cell carcinoma }\end{array}$ & 2.39 & 0.90 & 200.80 & 6.79 & 10.36 & 3.01 \\
\hline Stage I + II & 2.14 & 0.73 & 150.10 & 2.10 & 9.74 & 2.89 \\
\hline Stage III + IV & 2.54 & 1.06 & 234.85 & 2.94 & 10.52 & 3.03 \\
\hline Benign tumor & $1.61 * *$ & $0.6 I^{*}$ & $154.65^{*}$ & $\mathrm{I} .87^{*}$ & 8.72 & 2.79 \\
\hline Healthy persons & $1.39 * *$ & $0.60 *$ & II3.49* & I.48* & 10.33 & 2.73 \\
\hline$P_{1}$ & $<0.001$ & $<0.001$ & $<0.001$ & $<0.001$ & 0.082 & 0.585 \\
\hline$P_{2}$ & $<0.001$ & $<0.001$ & $<0.001$ & $<0.001$ & 0.149 & 0.632 \\
\hline$P_{3}$ & 0.043 & $<0.001$ & 0.002 & $<0.001$ & - & - \\
\hline$P_{4}^{3}$ & $<0.001$ & 0.981 & 0.964 & 0.430 & - & - \\
\hline$P_{5}^{4}$ & $<0.001$ & 0.953 & 0.059 & 0.054 & - & - \\
\hline$P_{6}$ & $<0.001$ & $<0.001$ & $<0.001$ & $<0.001$ & - & - \\
\hline$P_{7}$ & $<0.001$ & $<0.001$ & $<0.001$ & $<0.001$ & - & - \\
\hline$P_{8}$ & 0.170 & 0.934 & 0.050 & 0.155 & - & - \\
\hline
\end{tabular}

Notes: The data were analyzed using Kruskal-Wallis nonparametric rank sum test, the comparison among groups was performed using Mann-Whitney $U$ test. The measurement data were analyzed using M. $P_{1}$ was the comparison result between patients with OSCC/OPSCC, benign tumor group, and healthy group. $P_{2}$ was the comparison result between early oral/oropharyngeal squamous cell carcinoma (stage I + II) group, patients with middle-late OSCC/OPSCC (stage III + IV), benign tumor group, and healthy people. $P_{3}$ was the comparison result between early and patients with middle-late OSCC/OPSCC. $P_{4}$ was the comparison result between the early group and benign tumor group. $P_{5}$ was the comparison result between the early stage group and health control group. $P_{6}$ was the comparison result between the middle-late group and benign tumor group. $P_{7}$ was the comparison result between the middle-late group and healthy control group. $P_{8}$ was the comparison result between the benign tumor group and healthy group. **showed comparison with the early group, $P$-value $<0.05$; *showed comparison with the middle-advanced stage, $P$-value $<0.05$.

Abbreviations: AFP, $\alpha$-fetoprotein; CEA, carcinoembryonic antigen; CA19-9, carbohydrate antigen 19-9; Cyfra2I-I, cytokeratin I 9 fragment; M, median; P, patients; OSCC/ OPSCC, oral/oropharyngeal squamous carcinoma; SCCAg, squamous cell carcinoma antigen. 


\section{Diagnostic cutoff levels, sensitivities, specificities, and accuracies of serum TMs for patients with OSCC/OPSCC}

The receiver operating characteristic of serum Cyfra21-1, SCCAg, ferritin, and CEA levels in OSCC/OPSCC patients are shown in Figure 1. The diagnostic cutoff levels, sensitivities, specificities, and accuracies of serum TMs in patients with OSCC/OPSCC are shown in Table 3. The results demonstrated that the specificity of Cyfra21-1 was the highest, significantly higher than that of SCCAg, ferritin, and CEA $(P<0.05)$. The specificity of ferritin was the lowest, significantly lower than that of Cyfra21-1, SCCAg, and CEA $(P<0.05)$. There was no significant difference between the specificities of SCCAg and CEA $(P>0.05)$. The sensitivities of SCCAg and ferritin were significantly higher than those of Cyfra21-1 and CEA $(P<0.05)$. The sensitivities showed no significant difference between SCCAg and ferritin, and between Cyfra21-1 and CEA ( $P>0.05)$. The accuracies showed no significant difference among Cyfra21-1, SCCAg, ferritin, and CEA $(P>0.05)$.

\section{Discussion}

Serum TM has shown great developmental prospect for the screening and diagnosis of malignant tumor. ${ }^{7,8}$ There were a few serum TMs aiming for the screening and diagnosis of patients with OSCC/OPSCC. The common six serum

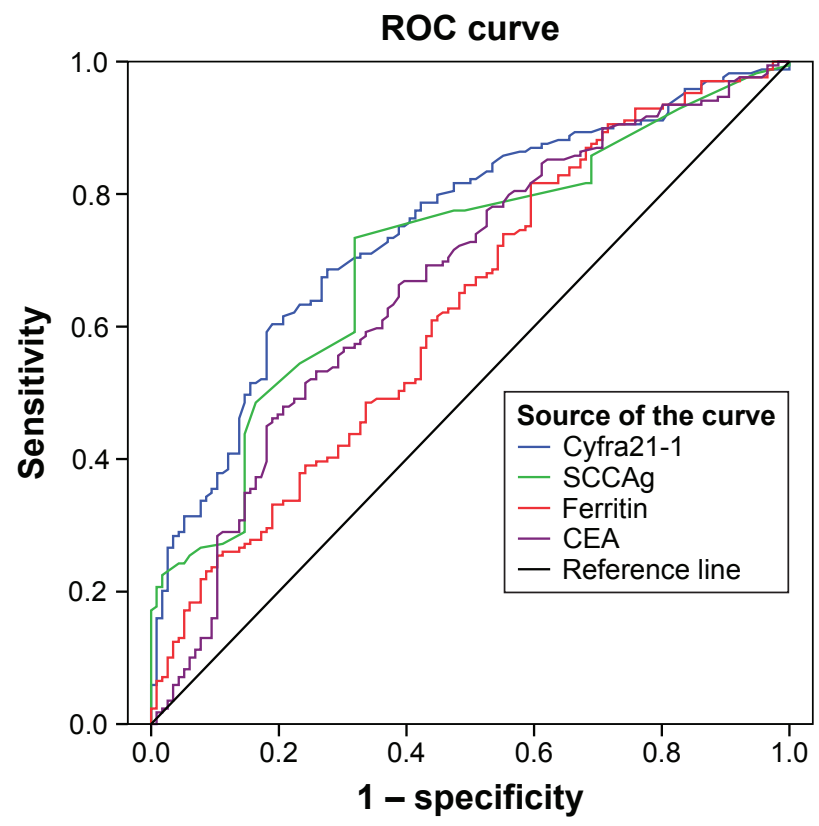

Figure I ROC of serum Cyfra2I-I, SCCAg, ferritin, and CEA for patients with OSCC/OPSCC.

Abbreviations: CEA, carcinoembryonic antigen; Cyfra2I-I, cytokeratin 19 fragment; OSCC/OPSCC, oral/oropharyngeal squamous carcinoma; ROC, receiver operating characteristic; SCCAg, squamous cell carcinoma antigen.
Table 3 Diagnostic cutoff levels, sensitivities, specificities, and accuracies of Cyfra2I-I, SCCAg, ferritin, CEA, CAI9-9, and AFP in oral/oropharyngeal squamous cell carcinoma

\begin{tabular}{lllll}
\hline Items & $\begin{array}{l}\text { Diagnostic } \\
\text { cutoff levels }\end{array}$ & Sensitivity \% & Specificity \% & Accuracy \% \\
\hline Cyfra2I-I & 2.17 & 60.36 & 81.03 & 68.77 \\
SCCAg & 0.72 & 73.37 & 68.10 & 71.23 \\
Ferritin & 109.95 & 81.66 & 40.52 & 64.91 \\
CEA & 1.99 & 66.27 & 61.21 & 64.21 \\
P-value & - & $<0.001$ & $<0.001$ & 0.236 \\
\hline
\end{tabular}

Notes: "-" shows no corresponding value. Diagnostic cutoff levels were calculated from the corresponding value of a point in the ROC curve, where the sum of the sensitivities and specificities was the largest value.

Abbreviations: AFP, $\alpha$-fetoprotein; CA19-9, carbohydrate antigen 19-9; CEA, carcinoembryonic antigen; Cyfra2 I-I, cytokeratin 19 fragment; SCCAg, squamous cell carcinoma antigen; ROC, receiver operating characteristic.

TMs were detected simultaneously first in this study, so as to analyze their diagnostic value for OSCC/OPSCC. The overall results suggested that the levels of serum CA19-9 and AFP showed no significant difference between patients with OSCC/OPSCC, benign tumor group, and healthy control group. So CA19-9 and AFP had no diagnostic value for patients with OSCC/OPSCC. The levels of serum Cyfra21-1, SCCAg, ferritin, and CEA were significantly increased in patients with OSCC/OPSCC, suggesting that they had a certain diagnostic value for patients with OSCC/OPSCC.

The ideal serum TMs should have higher sensitivity and specificity at the same time. Higher sensitivity often leads to lower specificity, and vice versa. Therefore, it is important to rationally confirm the diagnostic cutoff levels of the TMs. In this study, the optimal diagnostic cutoff level, corresponding sensitivity, and specificity of the TMs were obtained by receiver operating characteristic analysis. The research results showed that the sensitivities of SCCAg and ferritin were the highest and showed no significant difference. The specificities of SCCAg and ferritin were $68.10 \%$ and $40.52 \%$, respectively; the specificity of SCCAg was significantly higher than that of ferritin. Therefore, SCCAg had higher diagnostic value for patients with OSCC/OPSCC than that of ferritin. In the study, the specificity of Cyfra21-1 was the highest $(81.03 \%)$, the sensitivity was $60.36 \%$ and showed no significant difference compared to CEA. Overall, Cyfra21-1 and SCCAg had higher sensitivity and specificity. Therefore, the diagnostic values of Cyfra21-1 and SCCAg were superior to those of other TMs for patients with OSCC/OPSCC.

Cyfra21-1 is a soluble fragment of cytokeratin19 (CK19). CK19 fragment exists in the form of oligomer under normal circumstances. Its content is extremely low. If carcinogenesis occurs, the carcinoma cells could release Cyfra21-1 soluble fragments into blood circulation. ${ }^{15}$ Zhong et $\mathrm{al}^{22}$ reported 
that the sensitivity and specificity of Cyfra $21-1$ were $57 \%$ and $96.4 \%$ in patients with OSCC/OPSCC measured by enzyme-linked immunosorbent assay. The electrochemiluminescence immunoassay assay showed that the sensitivity and specificity of Cyfra $21-1$ were $60.36 \%$ and $81.03 \%$ in patients with OSCC/OPSCC in this study. The difference might be caused by different detection methods. Recently, Wang et $\mathrm{al}^{23}$ reported that the accuracy of serum Cyfra21-1 by enzyme-linked immunosorbent assay was superior to that of electrochemiluminescence immunoassay. In our study, patients with OSCC/OPSCC were divided into the early- and middle-late stage groups. The result showed that the level of serum Cyfra21-1 in patients with early OSCC/OPSCC was significantly higher than that of benign tumor group and healthy control group, suggesting that Cyfra21-1 had better clinical value in the screening and diagnosis of patients with early OSCC/OPSCC.

SCCAg is a subunit of squamous epithelial cell associated antigen TA-4, which is separated from cervical squamous epithelial cells. ${ }^{16}$ Kimura et $\mathrm{al}^{24}$ demonstrated that the level of serum SCCAg was increased in esophageal squamous cell carcinoma, anal squamous carcinoma, lung squamous cell carcinoma, and head and neck squamous cell carcinoma, which might have an auxiliary diagnostic value on the tumor. Feng et $\mathrm{al}^{25}$ showed that the concentration of serum SCCAg in oral squamous cell carcinoma patients was significantly higher than that of healthy control group; the sensitivity and specificity of SCCAg for oral squamous cell carcinoma were $51.40 \%$ and $73.60 \%$, respectively. The results of this study showed that the sensitivity and specificity of SCCAg were $73.37 \%$ and $68.10 \%$ in patients with OSCC/OPSCC. Further analysis showed that although the level of serum SCCAg in patients with OSCC/OPSCC was significantly higher than that of benign tumor group and healthy control group, the level of serum SCCAg showed no significant difference between patients with early OSCC/OPSCC, benign tumor group, and healthy control group, suggesting that the diagnostic value of SCCAg was poorer in patients with early OSCC/OPSCC.

\section{Conclusion}

In summary, among Cyfra21-1, SCCAg, ferritin, CEA, CA19-9, and AFP six serum TMs, Cyfra21-1 and SCCAg had higher sensitivity and specificity at the same time, their diagnoses for patients with OSCC/OPSCC were superior to those of other TMs, Cyfra21-1 had better clinical value for patients with early OSCC/OPSCC, and CA19-9 and AFP had no auxiliary diagnostic value for patients with OSCC/OPSCC.

\section{Disclosure}

The authors report no conflicts of interest in this work.

\section{References}

1. Van der Waal I. Are we able to reduce the mortality and morbidity of oral cancer; some considerations. Med Oral Pathol Oral Cir Bucal. 2013; 18(1):e33-e37.

2. Katz J, Jakymiw A, Ducksworth MK, et al. CIP2A expression and localization in oral carcinoma and dysplasia. Cancer Biol Ther. 2010; 10(7):694-699.

3. Marcos CA, Martinez DA, de Los Toyos JR, et al. The usefulness of new serum tumor markers in head and neck squamous cell carcinoma. Otolaryngol Head Neck Surg. 2009;140(3):375-380.

4. Warnakulasuriya S. Global epidemiology of oral and oropharyneal cancer. Oral Oncol. 2009;45(4-5):309-316.

5. Francisco AL, Correr WR, Pinto CA, et al. Analysis of surgical margins in oral cancer using in situ fluorescence spectroscopy. Oral Oncol. 2014;50(6):593-599.

6. Lee HJ, Kang YH, Lee JS, et al. Positive expression of NANOG, mutant p53, and CD44 is directly associated with clinicopathological features and poor prognosis of oral squamous cell carcinoma. BMC Oral Health. 2015;15(1): 153 .

7. Sargeran K, Murtomaa H, Safavi SM, Vehkalahti MM, Teronen O. Survival after diagnosis of cancer of the oral cavity. Br J Oral Maxillofac Surg. 2008;46(3):187-191.

8. Yonezawa K, Nishiumi S, Kitamoto-Matsuda J, et al. Serum and tissue metabolomics of head and neck cancer. Cancer Genomics Proteomics. 2013;10(5):233-238.

9. Rai NP, Anekar J, Shivaraja Shankara YM, et al. Comparison of serum fucose level in leukoplakia and oral cancer patients. Asian Pac J Cancer Prev. 2015;16(17):7497-7500.

10. Wang M, Devarajan K, Singal AG, et al. The Doylestown algorithm: a test to improve the performance of AFP in the detection of hepatocellular carcinoma. Cancer Prev Res (Phila). 2016;9(2):172-179.

11. Felgueiras J, Silva JV, Fardilha M. Prostate cancer: the need for biomarkers and new therapeutic targets. JZhejiang Univ Sci B. 2014;15(1): $16-42$.

12. Gadducci A, Tana R, Cosio S, Genazzani AR. The serum assay of tumour markers in the prognostic evaluation, treatment monitoring and follow-up of patients with cervical cancer: a review of the literature. Crit Rev Oncol Hematol. 2008;66(1):10-20.

13. Xu RH, Liao CZ, Luo Y, et al. Optimal cut-off values for CYFRA 21-1 expression in NSCLC patients depend on the presence of benign pulmonary diseases. Clin Chim Acta. 2015;440:188-192.

14. Huang C, Zhan T, Liu Y, et al. Glycomic profiling of carcinoembryonic antigen isolated from human tumor tissue. Clin Proteomics. 2015; 12(1):17.

15. Liu L, Liu B, Zhu LL, Li Y. CYFRA21-1 as a serum tumor marker for follow-up patients with squamous cell lung carcinoma and oropharynx squamous cell carcinoma. Biomark Med. 2013;7(4):591-599.

16. Kulpa J, Stasik Z, Skolyszewski J, Wójcik E, Rychlik U, Pudelek K. Predictive value of SCC-Ag, CYFRA 21-1 and selected acute phase proteins in radiotherapy of pharyngeal and laryngeal cancer. A preliminary report. Neoplasma. 2004;51(2):103-109.

17. Gwak HK, Lee JH, Park SG. Preliminary evaluation of clinical utility of CYFRA 21-1, CA 72-4, NSE, CA19-9 and CEA in stomach cancer. Asian Pac J Cancer Prev. 2014;15(12):4933-4938.

18. Barak V, Meirovitz A, Leibovici V, et al. The diagnostic and prognostic value of tumor markers (CEA, SCC, CYFRA21-1,TPS) in head and neck cancer patients. Anticancer Res. 2015;35(10):5519-5524.

19. Wang SL, Cao S, Wu R, et al. Serum ferritin predicted prognosis in patients with locally advanced pancreatic cancer. Future Oncol. 2015; 11(21):2905-2910.

20. Gui JC, Yan WL, Liu XD. CA19-9 and CA242 as tumor markers for the diagnosis of pancreatic cancer: a meta-analysis. Clin Exp Med. 2014;14(2):225-233. 
21. Okamura K, Takayama K, Izumi M, Harada T, Furuyama K, Nakanishi Y. Diagnostic value of CEA and CYFRA21-1 tumor markers in primary lung cancer. Lung Cancer. 2013;80(1):45-49.

22. Zhong LP, Zhu HG, Zhang CP, Chen WT, Zhang ZY. Detection of serum Cyfra 21-1 in patients with primary oral squamous cell carcinoma. Int J Oral Maxillofac Surg. 2007;36(3):230-234.

23. Wang YX, Hu D, Yan X. Diagnostic accuracy of Cyfra 21-1 for head and neck squamous cell carcinoma: a meta-analysis. Eur Rev Med Pharmacol Sci. 2013;17(17):2383-2389.
24. Kimura Y, Fujieda S, Takabayashi T, Tanaka T, Suqimoto C, Saito H. Conventional tumor markers are prognostic indicators in patients with head and neck squamous cell carcinoma. Cancer Lett. 2000; 155(2):163-168.

25. Feng XY, Li JH, Li JZ, Han ZX, Xing RD. Serum SCCA, Cyfra 21-1, EGFR and Cyclin D1 levels in patients with oral squamous cell carcinoma. Int J Biol Markers. 2010;25(2):93-98.

\section{Publish your work in this journal}

OncoTargets and Therapy is an international, peer-reviewed, open access journal focusing on the pathological basis of all cancers, potential targets for therapy and treatment protocols employed to improve the management of cancer patients. The journal also focuses on the impact of management programs and new therapeutic agents and protocols on

patient perspectives such as quality of life, adherence and satisfaction. The manuscript management system is completely online and includes a very quick and fair peer-review system, which is all easy to use. Visit http://www.dovepress.com/testimonials.php to read real quotes from published authors. 